\title{
Model of Healthcare-Associated Infection Control in Primary Health Care Institutions: A Structural Equation Modeling*
}

\author{
Zi-nan ZHANG ${ }^{1}$, Xin-ping ZHANG ${ }^{1 \#}$, Xiao-quan $\mathrm{LAI}^{2 \#}$ \\ ${ }^{1}$ School of Medicine and Health Management, Tongji Medical College, Huazhong University of Science and Technology, \\ Wuhan 430030, China \\ ${ }^{2}$ Department of Nosocomial Infection, Tongji Hospital, Tongji Medical College, Huazhong University of Science and Technology, \\ Wuhan 430030, China
}

(C) Huazhong University of Science and Technology 2019

\begin{abstract}
Summary: The purpose of this study was to construct the model of organization system, management, training and surveillance in healthcare-associated infection prevention and control (IC) of primary health care institutions and identify its effect on patient safety and decreasing economic burden by standardizing IC. A cross-sectional survey was conducted with questionnaires. Data were collected from 268 primary health care institutions in Hubei province, China. Hypotheses on the model of IC were analyzed by means of confirmatory factor analysis and structural equation modeling. The results showed that the fit indices of the hypothesized model of IC satisfied recommended levels: root mean square error of approximation (RMSEA) $=0.071$; comparative fit index $(\mathrm{CFI})=0.965$; tucker-lewis index $(\mathrm{TLI})=0.956$; weighted root mean square residual $(\mathrm{WRMR})=1.014$. The model showed that organization system had a direct effect on management $(\beta=0.311, P<0.01)$, and training $(\beta=0.365, P<0.01)$. Management and training played an intermediary role that partially promoted organization system impact on surveillance. Results also showed that institutional factors such as the number of physicians, the number of nurses, the designated capacity of beds, the actual number of open beds and surgery trips had positive impacts on management $(\beta=0.050, P<0.01 ; \beta=0.181, P<0.01 ; \beta=0.111, P<0.01 ; \beta=0.064, P<0.01 ; \beta=0.084$, $P=0.04)$ and training $(\beta=0.21, P=0.03 ; \beta=0.050, P=0.02 ; \beta=0.586, P=0.01 ; \beta=0.995, P=0.02$; $\beta=-0.223, P=0.03)$. In conclusion, the model of organization system, management, training and surveillance in IC of primary health care institutions is valuable for guiding IC practice.

Key words: model; organization system; management; training; surveillance; infection control; primary health care institutions
\end{abstract}

The primary health care system in China, which provides basic clinical care and public health services to a fifth of the world's population, has a notable history. In the past decades, these primary care institutions faced substantial challenges after market-based reforms in the health care sector, including limited development, inadequate government funding and weakening of the support by public health care providers ${ }^{[1]}$. This led to unintended consequences such as surging costs, poor quality, diminished access to care and brain drain in primary care institutions ${ }^{[2-4]}$. Even though China has made remarkable efforts and achievements since 2009, the service quality of primary health care in China is poor. Some evidence points the substantial gaps

Zi-nan ZHANG, E-mail: zinan19880609@126.com ${ }^{\#}$ Corresponding authors, Xin-ping ZHANG, E-mail: xpzhang602@hust.edu.cn; Xiao-quan LAI, E-mail: 3057606997@qq.com

*This study was supported by the National Natural Science Foundation of China (No. 71473098). between the quality of processes and outcomes ${ }^{[5]}$.

Healthcare-associated infection (HAI) in the quality improvement is responsible for a substantial burden of diseases on patients and healthcare workers, especially in primary health care institutions with poor resources ${ }^{[6]}$. For these institutions, poor social environment, deficient infrastructures, rudimentary equipment, the lack of national and local infection control policies and the co-existence of other major health problems are the main determinants of the poor quality of care and a two to twenty times higher risk of acquiring $\mathrm{HAI}^{[7]}$. In developing countries, surveillance systems providing reliable and regularly collected data are non-existent and the burden of HAI is underestimated to a large extent and unknown by healthcare professionals and policy makers. Data from some researches clearly indicate that surveillance system is a major hidden problem affecting patients both psycho-physically and economically, as well as affecting healthcare workers ${ }^{[8]}$. 
The infection control (IC) should be valued in the entire service process. Any problems happened during the service process can lead to $\mathrm{HAI}^{[9,10]}$. According to the relevant laws and regulations promulgated by the National Health and Family Planning Commission ${ }^{[11]}$, the corresponding organization system must be set up, and management, training and surveillance should be strengthened. Many countries with efficient IC management also attach great importance to the construction of IC organization and management ${ }^{[12]}$, with particular emphasis on IC training and HAI surveillance $^{[13]}$. Limited researches have shown the importance of organization system and management. The HAI rate can be reduced effectively and the compliance rate of health workers can be improved in the daily work by the efficient management. According to a research of Menegueti ${ }^{[14]}$, in the developing countries, although there were relevant laws and regulations of IC, the evaluation system was deficient, and the problem of IC was not well solved. Thus, indicators of management program were important parts to evaluate IC mode.

At present, there are limited researches on the management of primary health care institutions, especially the quantitative research of IC process indicators ${ }^{[15]}$, such as organization system and management. For primary health care institutions, researches associated with human resources, equipment configuration and process indicators of IC are particularly important.

This study aimed to examine the IC organization system and management mode, and explore the role of IC organization system and management mode in the standardization of primary health care institutions, as well as impacting factors for IC management. The results provide an important evidence-based value for the construction and management of IC organization system in primary health care institutions. Under the primary health policy and the national strategy for countryside, it is of great theoretical and practical significance to study the influence of the size and IC organization system and management mode on primary health care institutions.

\section{METHODS}

\subsection{Conceptual Framework}

Conceptual framework of this study was constructed based on a review of previous studies on HAI control. Prevention and control of HAI depended on high levels of organization system ${ }^{[9,12]}$. Effective management strategies and plans were related to rational organization system ${ }^{[9,}{ }^{10]}$. In addition, training of personnel skills was impacted by organization system such as leadership of institutions ${ }^{[9,14]}$. The surveillance system and feedback of surveillance matters depended on rational organization system ${ }^{[9,10]}$. Surveillance matters were related to the effective management strategies and plans ${ }^{[9,16]}$. The emphasis of HAI during the training for staff of institutions benefited for surveillance ${ }^{[14]}$. And the good implementation of plans $\&$ strategies was beneficial to surveillance ${ }^{[9]}$.

Six hypotheses were included in this study (fig. 1). Organization system has positive impact on management (H1). Organization system has positive impact on training (H2). Organization system has positive impact on surveillance (H3). Management has positive impact on training (H4). Training has positive impact on surveillance (H5). Management is positively related to surveillance (H6).

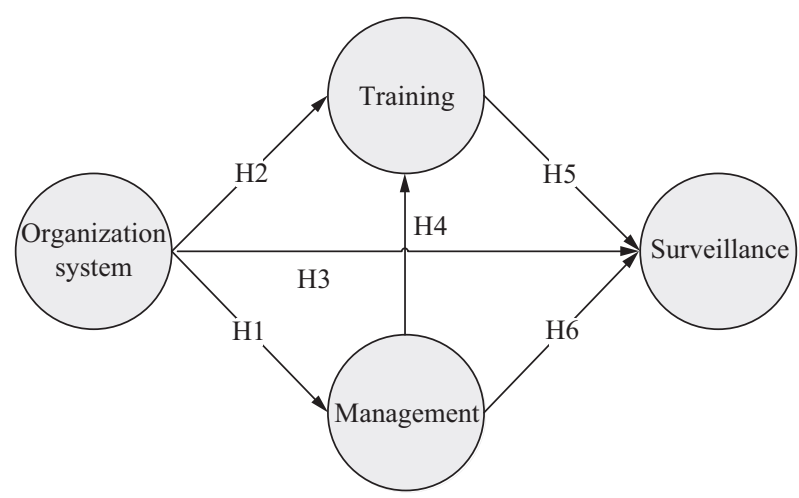

Fig. 1 Hypothesized model framework of organization system, management, training and surveillance on IC

\subsection{Questionnaire Survey}

The primary health care institutions, including township health centers and community health centers were selected as study objects. The cluster sampling was performed from the primary health care institutions in 13 cities located in Hubei province. A questionnaire survey was conducted to collect the information of the primary health care institutions, including organization system, management, training and surveillance on IC according to the related research, together with the factors of the primary health care institutions such as the number of physicians, nurses and beds, etc..

\subsection{Latent and Observed Variables}

The latent variables of this study were composed of institutions, organization system, management, training and surveillance. Observed variables measuring the organization system included IC responsibility of professionals (A1, categorical variable), the director as first responsibility for IC (A2, categorical variable), IC professionals (A3, categorical variable), the number of IC part-time staff (A4, continuous variable), the number of IC certificate for professionals (A5, continuous variable), IC staffing needs (A6, continuous variable) and other 12 variables. Observed variables measuring the management included the form of making IC annual plan (B1, categorical variable), the implementation of IC annual plan (B2, categorical variable), frequency 
of evaluating objectives (per year) (B3, continuous variable), completeness of IC evaluation report (B4, categorical variable), regular monitoring and guidance on IC (B5, categorical variable), the subject of regular monitoring and guidance on IC (B6, categorical variable), standard procedure for regular monitoring and guidance on IC (B7, categorical variable), the number of regular monitoring and guidance on IC (B8, continuous variable) and other 13 variables. Observed variables measuring the training included the number of IC staff training ( $\mathrm{C} 1$, continuous variable), the notice of IC training ( $\mathrm{C} 2$, categorical variable), the material of IC training (C3, categorical variable), multimedia material of IC training ( $\mathrm{C} 4$, categorical variable), the summary of IC training (C5, categorical variable) and other 10 variables. Observed variables measuring the surveillance included case surveillance of HAI (D1, categorical variable), surveillance on environmental hygiene (D2, categorical variable), surveillance on effect of disinfection and sterilization (D3, categorical variable) and other 7 variables. Above all, 42 observed variables were included in the questionnaire.

Factors associated with institutions were composed of the number of physicians, the number of nurses, the designated capacity of beds, the actual number of open beds, outpatient visits, inpatient visits and surgical trips.

\subsection{Statistical Analysis}

A Structural Equation Modelling (SEM) approach was performed by using weighted least squares with mean and variance adjusted (WLSMV). The WLSMV estimation approach was designed specifically to handle categorical variables and yielded good estimates of the parameters for both non-normal category data and small sample data ${ }^{[17]}$. The following criteria were used to identify problematic items: (1) cross loading $(>0.3)$ on a factor other than the hypothesized factor; (2) cross loading $(>0.2)$ on two or more other factors. The SEM model fit criteria used were: root mean square error of approximation (RMSEA) $<0.08$, weighted root mean square Residual (WRMR) $<1$, comparative fit index (CFI) $>0.90$, Tucker-Lewis index $(\mathrm{TLI})>0.90^{[17-20]}$. SPSS version 17 and Mplus version 7 were used.

\section{RESULTS}

\subsection{Size of Primary Health Care Institutions}

Totally, 308 primary health care institutions were selected from 13 prefectural-level cities in Hubei province, China, with 268 qualified institutions which made the pass rate of $87 \%$. The number of responding physicians was $18.14 \pm 14.26$, and $39.6 \%$ had fewer than 10 . The number of responding nurses was $18.45 \pm 13.78$, and $34 \%$ had between 11 and 20 . The designated capacity of beds was $54.53 \pm 31.67$, and $95.8 \%$ had fewer than 100 . The actual number of open beds was $59.05 \pm 43.73$, and $88.5 \%$ had fewer than 100 . The number of outpatient visits was $2222.85 \pm 1071.32$. The number of inpatient visits was $2410.83 \pm 2043.72$. The number of surgery trips was $184.02 \pm 113.97$.

\subsection{Model of Organization System, Management, Training and Surveillance on IC}

The model of organization system, management, training and surveillance on IC was constructed based on 22 observed variables after 20 observed variables were excluded based on pre-specified criteria (see Section 1.3).

The model of fitness was as follows: RMSEA $=$ 0.071; $\mathrm{CFI}=0.965 ; \mathrm{TLI}=0.956$; $\mathrm{WRMR}=1.014$ with no correlated errors or cross-loadings. All the fit indices met the recommended level (fig. 2). The model on IC had a good fit.

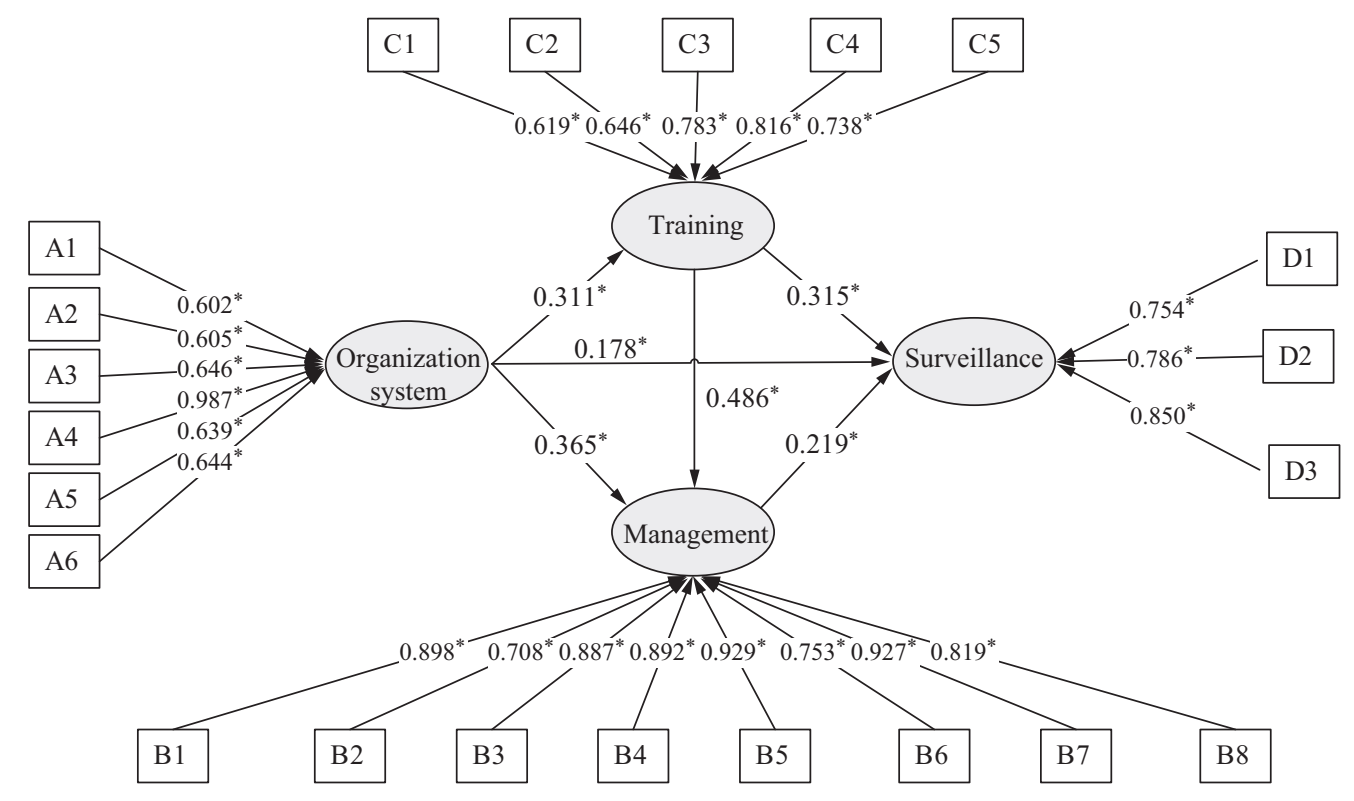

Fig. 2 Structural equation modeling of organization system, management, training and surveillance on IC 
Table 1 Direct and indirect effects of study variables for the final model structure paths

\begin{tabular}{lccc}
\hline Paths & Standardized estimate & S.E. & Two-tailed $P$ value \\
\hline Organization system $\rightarrow$ Management & 0.311 & 0.088 & $<0.01$ \\
Organization system $\rightarrow$ Training & 0.365 & 0.103 & $<0.01$ \\
Management $\rightarrow$ Surveillance & 0.315 & 0.125 & 0.012 \\
Training $\rightarrow$ Surveillance & 0.219 & 0.111 & 0.048 \\
Training $\rightarrow$ Management & 0.486 & 0.071 & $<0.01$ \\
Organization system $\rightarrow$ Surveillance & 0.178 & 0.053 & 0.001 \\
Organization system $\rightarrow$ Training $\rightarrow$ Surveillance & 0.071 & 0.042 & 0.044 \\
Organization system $\rightarrow$ Management $\rightarrow$ Surveillance & 0.087 & 0.043 & 0.036 \\
\hline
\end{tabular}

Table 1 showed the relationship of latent variables for the final model. Organization system manifested direct effects on management $(\beta=0.311$, $P<0.01)$, training $(\beta=0.365, P<0.01)$, surveillance $(\beta=0.178, P<0.01)$ respectively, and an indirect effect on surveillance through management and training ( $\beta=0.071, P=0.044 ; \beta=0.087, P=0.0036)$. Management $(\beta=0.315, P=0.012)$ and training $(\beta=0.219, P=0.048)$ had direct effects on surveillance. Training $(\beta=0.486$, $P=0.048$ ) had direct effects on management.

Direct and indirect effects of study variables for the final model structure paths: The final model with 4 latent variables had a good fit. There were interactions between 4 latent variables: organization system had a direct positive effect on management, training and surveillance. The standardized path coefficients of each direct effect were $0.311,0.355$ and 0.178 respectively. Management and training also had a direct positive effect on surveillance. The standardized path coefficients of each direct effect were 0.315 and 0.219 respectively. Training also had a direct positive effect on management with a standardized path coefficient of 0.486 . At the same time, the organization system also indirectly influenced the surveillance through management and training. The standardized path coefficients of indirect effects were 0.087 and 0.071 , respectively. According to the direct and indirect effects of all factors, it could be seen that the management, training and surveillance had been improved by $31.1 \%$, $36.5 \%$ and $33.6 \%$ respectively when the organization system improved its degree of perfection by $1 \%$; when the management level was increased by $1 \%$, its surveillance level could be increased by $31.5 \%$; when the level of training was increased by $1 \%$, its surveillance level could be increased by $21.9 \%$ and its organization system and management level increased by $48.6 \%$.

\subsection{Impact of Size of Institutions and Latent Variables on IC Model}

The number of physicians had direct effects on management $(\beta=0.05, P<0.01)$. The number of nurses had direct effects on management $(\beta=0.181, P<0.01)$. The designated capacity of beds had direct effects on management $(\beta=0.111, P<0.01)$. The actual number of open beds had direct effects on management $(\beta=0.064, P<0.01)$. Surgery trips had direct effects on management $(\beta=0.084, P=0.04)$. The number of physicians had direct effects on training $(\beta=0.21$, $P=0.03)$. The number of nurses had direct effects on training $(\beta=0.05, P=0.02)$. The designated capacity of beds had direct effects on training $(\beta=0.586, P=0.01)$. The actual number of open beds had direct effects on training $(\beta=0.995, P=0.02)$. Surgery trips had direct effects on training $(\beta=0.223, P=0.034)$ (table 2$)$.

\section{DISCUSSION}

The model of organization system, management, training and surveillance on IC in Primary Health Care Institutions was constructed based on 22 observed variables. And all hypotheses in the model were tested and accepted except for hypothesis 4 (H4), which implied that IC management in primary health care institutions has guiding and referencing value for future reform.

Most of the results related to the relationship

Table 2 Impact of size of institutions and latent variables on IC model

\begin{tabular}{|c|c|c|c|c|c|c|c|c|c|c|c|c|}
\hline \multirow{2}{*}{ Variables } & \multicolumn{3}{|c|}{ Management } & \multicolumn{3}{|c|}{ Training } & \multicolumn{3}{|c|}{ Organization system } & \multicolumn{3}{|c|}{ Surveillance } \\
\hline & $E$ & S.E. & $P$ & $E$ & S.E. & $P$ & $E$ & S.E. & $P$ & $E$ & S.E. & $P$ \\
\hline Number of physicians & 0.050 & 0.010 & $<0.01$ & 0.210 & 0.008 & 0.03 & -0.015 & 0.026 & 0.575 & -0.002 & 0.012 & 0.875 \\
\hline Number of nurses & 0.181 & 0.010 & $<0.01$ & 0.050 & 0.011 & 0.02 & -0.004 & 0.037 & 0.917 & 0.003 & 0.012 & 0.796 \\
\hline Number of designated beds & 0.111 & 0.003 & $<0.01$ & 0.586 & 0.012 & 0.01 & -0.006 & 0.007 & 0.401 & 0.000 & 0.003 & 0.962 \\
\hline Number of open beds & 0.064 & 0.002 & $<0.01$ & -0.995 & 0.003 & 0.02 & 0.000 & 0.001 & 0.644 & 0.000 & 0.000 & 0.920 \\
\hline Outpatient visits & 0.110 & 0.001 & 0.58 & -0.008 & 0.001 & 0.97 & 0.000 & 0.000 & 0.971 & 0.000 & 0.000 & 0.395 \\
\hline Inpatient visits & -0.076 & 0.001 & 0.56 & 0.067 & 0.002 & 0.43 & 0.000 & 0.000 & 0.420 & 0.000 & 0.000 & 0.395 \\
\hline Surgery trips & 0.084 & 0.001 & 0.04 & -0.223 & 0.001 & 0.03 & -0.001 & 0.001 & 0.527 & 0.000 & 0.001 & 0.613 \\
\hline
\end{tabular}

E: Estimate; $P$ : two-tailed $P$-value 
of latent variables in the model are consistent with the previous researches. The degree of organization system integrity determines the development of IC management, training and surveillance ${ }^{[9,10,21]}$. In terms of $\mathrm{H} 4$, some results of previous studies are consistent with our findings. Research in hand hygiene compliance and safe injection also shows that training of ICrelated knowledge throughout the organization system can increase the operating standardization of health workers in daily work $^{[13,14,22-24]}$. Strengthening the training of health workers and improving the capability of HAI management can promote IC surveillance activities $^{[16,25]}$. Surveillance is an important impact factor of IC. However, the situation of lacking management strategy for IC workers is the main reason for bad result. On the other hand, the bad result is also caused by the fact that IC workers do not comply with the relevant regulations. A similar research had found that policy makers did not have enough knowledge about IC, lack the effective surveillance, and the outcome of IC was limit ${ }^{[26]}$. Another study in developing country showed that the epidemiological surveillance data were not sent to the commission by IC workers, and they did only select these data, resulting in the ineffective interventions of $\mathrm{IC}^{[12]}$. Similar conclusions can be drawn from these studies. The training of health workers may standardize their IC behavior, increase their attention and promote them to carry out corresponding surveillance activities, which can ultimately reduce the HAI rate ${ }^{[23]}$.

The institution related factors have an impact on organization system and management. The study suggested that the number of physicians and nurses, the designated capacity of beds, the actual number of open beds and surgical visits in 5 years had a significant effect on the management. The results of standardized regression coefficients showed that the sizes of institutions have positive impact on the management ${ }^{[9]}$. Zingg et al pointed out that a decrease in the number of physicians and nurses was associated with an increase in HAI rates ${ }^{[9]}$. They suggested that all health care facilities should be adequately staffed according to the ratio of doctors and nurses, particularly those such as ICU, neonatal district and operating room. And there must be equipped sufficient personnel to complete the basic IC work $^{[12]}$. The study by Padoveze et al concluded that the management work was extremely terrible at medical institutions with less than 50 beds in Brazil, and the reason for this analysis was mainly due to inadequate IC full-time staffing ${ }^{[27]}$. No relevant research has been reported on the surgical visits effects. Based on the data from this study, organization system with more people and with more operations are more likely to have better bed space management and medical staffing than those with the above two influencing factors ${ }^{[11]}$.
The institution related factors also have an impact on training. This study found that the number of physicians, nurses, the designated capacity of beds, the actual number of open beds and surgical visits in 5 years had significant effects on the training, while the actual number of open beds and surgical visits on the training were negative. That is, both of the increases in the actual number of open beds and surgical visits can lead to a decline in the quality of training. Similar studies had shown that job burnout was a major contributing factor to decline in the quality of training ${ }^{[28-30]}$. Especially in the low-income and middle-income countries and regions, similar research results showed that there were more such situations in the operating room, ICU and other departments, which were caused by the higher intensity and lower income of health care workers of IC who were less compliant and less motivated to conduct IC measures and training ${ }^{[28]}$.

This study also found that sizes of institutions have no impact on organization system and surveillance. No research had so far given definitive conclusions regarding the size of the institutions and the establishment of a matching organization system of IC, especially in the area of primary health care institutions ${ }^{[12,31]}$. Based on the results of this study, the reason may be that the research objects are primary health care institutions with small number of staff, fewer personnel engaged in HAI, and insufficient data to reflect the impact on the organization system. This study has some limitations. First, this study was conducted in Hubei province, and the findings should be carefully generalized. The status quo in other provinces of China may need to be addressed in a future study. Second, the results of the survey could be more excellent than the actual conditions, which might be contributed to the fact that the staff of primary health care institutions tended to do their best when they knew that they were being observed.

To sum up, first, our findings show that organization system is a priority for HAI control in primary health care institutions. Management, training and surveillance are directly impacted by organization system of IC. In addition, the influences of training on the implementation of HAI strategy are particularly important in primary health care institutions. Finally, the important factors that affect management and training of IC include the number of physicians, the number of nurses, the designated capacity of beds, the actual number of open beds and surgical visits in the medical institutions. These findings suggest that sound organization system, accentuation of training among health care workers and optimal staffing allocation are necessary to improve IC in primary health care institutions.

\section{Acknowledgements}

The authors would like to thank Li-ping YE, Chen-xi 
LIU, Meng-hui ZHANG, Hai-hong CHEN, Dan WANG, Jun-jie LIU, Yan CHAO, Xin JU for their support of data collection and substantive suggestions for revision in the study.

\section{Conflict of Interest Statement}

We declare that we have no conflict of interest.

\section{REFERENCES}

1 Zhang L. The Chinese State's Retreat from Health: Policy and the Politics of Retrenchment. Routledge, 2012.

2 Hu S, Tang S, Liu Y, et al. Reform of how health care is paid for in China: challenges and opportunities. Lancet, 2008,372(9652):1846

3 Tang S, Meng Q, Chen L, et al. Tackling the challenges to health equity in China. Lancet, 2008,372(9648):14931501

4 Zhang D, Unschuld PU. China's barefoot doctor: past, present, and future. Lancet, 2008,372(9653):1865

$5 \mathrm{Li} \mathrm{X}, \mathrm{Lu} \mathrm{J}, \mathrm{Hu} \mathrm{S}$, et al. The primary health-care system in China. Lancet, 2017,390(10112):2584-2594

6 Harries AD, Zachariah R, Taylersmith K, et al. Keeping health facilities safe: one way of strengthening the interaction between disease-specific programmes and health systems. Trop Med Int Health, 2010,15(12):14071412

7 Pittet D, Allegranzi B, Storr J, et al. The Global Patient Safety Challenge 2005-2006: Clean Care is Safer Care. Int J Infect Dis, 2006,10(6):419-424

8 Zaidi AK, Huskins WC, Thaver D, et al. Hospitalacquired neonatal infections in developing countries. Lancet, 2005,365(9465):1175-1188

9 Zingg W, Holmes A, Dettenkofer M, et al. Hospital organisation, management, and structure for prevention of health-care-associated infection: a systematic review and expert consensus. Lancet Infect Dis, 2015,15(2):212224

10 Hansen S, Zingg W, Ahmad R, et al. Organizing of infection control in European hospitals. J Hosp Infect, 2015,91(4):338-345

11 National Health and Family Planning Commission. Regulation of Healthcare-Associated Infection Management (Chinese). 2006.

12 Rodríguez-Baño J, del Toro MD, López-Méndez J, et al. Minimum requirements in infection control. Clin Microbiol Infect, 2015,21(12):1072-1076

13 Yilmaz G, Aydin H, Aydin M, et al. Staff education aimed at reducing ventilator-associated pneumonia. J Med Microbiol, 2016,1378-1384

14 Menegueti MG, Canini SR, Bellissimo-Rodrigues $\mathrm{F}$, et al. Evaluation of nosocomial infection control programs in health services. Rev Lat Am Enfermagem, 2015,23(1):98-105

15 Allegranzi B, Nejad SB, Combescure C, et al. Burden of endemic health-care-associated infection in developing countries: systematic review and meta-analysis. Lancet, 2011,377(9761):228-241
16 Cheng VC, Tai JW, Wong LM, et al. Effect of proactive infection control measures on benchmarked rate of hospital outbreaks: An analysis of public hospitals in Hong Kong over 5 years. Am J Infect Control, 2015,43(9):965-970

17 Beauducel A, Herzberg PY. On the Performance of Maximum Likelihood Versus Means and Variance Adjusted Weighted Least Squares Estimation in CFA. Struct Equ Modeling, 2006,13(2):186-203

18 Schreiber JB, Stage FK, King J, et al. Reporting Structural Equation Modeling and Confirmatory Factor Analysis Results: A Review. J Educ Res, 2006,99(6):323-338

19 Hooper D, Coughlan J, Mullen MR. Structural equation modelling: Guidelines for determining model fit. Electronic J Business Res Methods, 2008,6(1):141-146

20 Barrett P. Structural equation modelling: Adjudging model fit. Pers Individ Differences, 2007,42(5):815-824

21 Mamishi S, Pourakbari B, Teymuri M, et al. Management of hospital infection control in Iran: a need for implementation of multidisciplinary approach. Osong Public Health Res Perspect, 2014,5(4):179-186

22 Bedoya G, Dolinger A, Rogo K, et al. Observations of infection prevention and control practices in primary health care, Kenya. Bull World Health Organ, 2017.

23 Brusaferro S, Arnoldo L, Cattani G, et al. Harmonizing and supporting infection control training in Europe. J Hosp Infect, 2015,89(4):351-356

24 Ye LP, Zhang XP, Lai XQ. Does Hospital Ownership Influence Hand Hygiene Compliance? J Huazhong Univ Sci Technolog Med Sci, 2017,37(5):787-794

25 Flanagan E, Chopra T, Mody L. Infection control in alternative healthcare settings. Infect Dis Clin North, 2016,30(3):785-804

26 Brannigan E, Murray E, Holmes A. Where does infection control fit into a hospital management structure? J Hosp Infect, 2009,73(4):392-396

27 Padoveze MC, Fortaleza CMCB, Kiffer C, et al. Structure for prevention of health care-associated infections in Brazilian hospitals: A countrywide study. Am J Infect Control, 2016,44(1):74-79

28 Colindres CV, Bryce E, Coral-Rosero P, et al. Effect of effort-reward imbalance and burnout on infection control among Ecuadorian nurses. Int Nurs Rev, 2018(2):190-199

29 Cheema AA, Scott AM, Shambaugh KJ, et al. Rebound in ventilator-associated pneumonia rates during a prevention checklist washout period. BMJ Qual Saf, 2011,20(9):811-817

30 Pratt M, Kerr M, Wong C. The impact of ERI, burnout, and caring for SARS patients on hospital nurses' selfreported compliance with infection control. Can J Infect Control, 2009,24(3):167-172

31 Murphy DM. Staffing and structure of infection prevention and control programs. Am J Infect Control, 2009,37(5):349-350

(Received Mar. 11, 2018; revised Jan. 9, 2019) 